\title{
The maximality principle revisited: on certain optimal stopping problems
}

\author{
Jan Obłój*
}

May 12, 2004

\begin{abstract}
We investigate in detail works of Peskir 13 and Meilijson 9 and develop a link between them. We consider the following optimal stopping problem: maximize $V_{\tau}=\mathbb{E}\left[\phi\left(S_{\tau}\right)-\int_{0}^{\tau} c\left(B_{s}\right) d s\right]$ over all stopping times with $\mathbb{E} \int_{0}^{\tau} c\left(B_{s}\right) d s<\infty$, where $S=\left(S_{t}\right)_{t \geq 0}$ is the supremum process associated with real valued Brownian motion $B, \phi \in C^{1}$ is non-decreasing and $c$ is continuous. From work of Peskir [13] we deduce that this problem has a unique solution if and only if the differential equation

$$
g^{\prime}(s)=\frac{\phi^{\prime}(s)}{2 c(g(s))(s-g(s))}
$$

admits a maximal solution $g_{*}(s)$ such that $g_{*}(s) \leq s$ for all $s \geq 0$. The stopping time which yields the highest payoff can be written as $\tau_{*}=$ $\inf \left\{t \geq 0: B_{t} \leq g_{*}\left(S_{t}\right)\right\}$. The problem is actually solved in a general case of a real-valued, time homogeneous diffusion $X=\left(X_{t}: t \geq 0\right)$ instead of $B$. We then proceed to solve the problem for more general functions $\phi$ and $c$. Explicit formulae for payoff are given.

We apply the results to solve the so-called optimal Skorokhod embedding problem. We give also a sample of applications to various inequalities dealing with terminal value and supremum of a process.
\end{abstract}

Keywords: Optimal stopping, maximality principle, optimal Skorokhod embedding, maximum process.

Mathematics Subject Classification: 60G40, $60 \mathrm{~J} 60$

\footnotetext{
* Laboratoire de Probabilités et Modèles Aléatoires, Université Paris 6 , 4 pl. Jussieu, Boite 188, 75252 Paris Cedex 05, France

Wydział Matematyki, Uniwersytet Warszawski

ul. Banacha 2, 02-097 Warszawa, Poland

email: obloj@mimuw.edu.pl

WWW: www.mimuw.edu.pl/ obloj/

Work partially supported by Polish Academy of Science scholarship.
} 


\section{Introduction}

Let $\phi$ be a non-negative, increasing, continuous function and $c$ a continuous, positive function, and consider the following optimal stopping problem of maximizing

$$
V_{\tau}=\mathbb{E}\left[\phi\left(S_{\tau}\right)-\int_{0}^{\tau} c\left(B_{s}\right) d s\right]
$$

over all stopping times $\tau$ such that

$$
\mathbb{E}\left[\int_{0}^{\tau} c\left(B_{s}\right) d s\right]<\infty
$$

Suppose, in the first moment, that $\phi(x)=x$ and $c(x)=c>0$ is a constant. In this formulation the problem was solved by Dubins and Schwarz [5] in an article on Doob-like inequalities. The optimal stopping time is just the Azéma-Yor embedding for a shifted (to be centered) exponential distribution (with parameter $2 c$ ). This leads in particular to an optimal inequality 6.1).

Keeping $\phi(x)=x$ let $c(x)$ be a non-negative, continuous function. The setup was treated by Peskir in a series of articles ([13], 14, 15]). He treated the case of real diffusions, which allows to recover the solution for general $\phi$ as a corollary. We want to maximize (1.1) over all stopping times $\tau$ satisfying (1.2).

Theorem 1 (Peskir [13]). The problem 1.1]), for $\phi(x)=x$ and $c(x)$ a non-negative, continuous function, has an optimal solution with finite payoff if and only if there exists a maximal solution $g_{*}$ of

$$
g^{\prime}(s)=\frac{1}{2 c(g(s))(s-g(s))}
$$

which stays strictly below the diagonal in $\mathbb{R}^{2}$. Azéma-Yor stopping time $\tau_{*}=\inf \left\{t \geq 0: B_{t} \leq g_{*}\left(S_{t}\right)\right\}$ is then optimal and satisfies (1.2) whenever there exists a stopping time which satisfies 1.2).

As pointed out above, this theorem was proved in a setup of any real, regular, time-homogenous diffusion to which we will come back later. The characterization of existence of a solution to (1.1) through existence of a solution to the differential equation (1.3) is called the maximality principle.

Let now $\phi$ be any non-negative, non-decreasing, right-continuous function such that $\phi\left(B_{t}\right)-c t$ is a.s. negative on some $\left(t_{0}, \infty\right)$ (with $t_{0}$ random) and keep $c$ constant. This optimal stopping problem was solved by Meilijson. Define

$$
H(x)=\sup _{\tau} \mathbb{E}\left[\phi\left(x+S_{\tau}\right)-c \tau\right] .
$$

Theorem 2 (Meilijson 9]). Suppose that $\mathbb{E} \sup _{t}\left\{\phi\left(B_{t}\right)-c t\right\}<\infty$. Then $H$ is absolutely continuous and is the minimal solution of the differential equation

$$
H(x)-\frac{1}{4 c}\left(H^{\prime}(x)\right)^{2}=\phi(x) .
$$

If $\phi$ is constant on $\left[x_{0}, \infty\right)$ then $H$ is the unique solution of (1.4) that equals $\phi$ on $\left[x_{0}, \infty\right)$. The optimal stopping time $\tau_{*}$ which yields $H(0)$ is the Azéma-Yor stopping time given by $\tau_{*}=\inf \left\{t \geq 0: B_{t} \leq S_{t}-\frac{H^{\prime}\left(S_{t}\right)}{2 c}\right\}$. 
Let us examine in more detail the result of Meilijson in order to compare it with the result of Peskir. The Azéma-Yor stopping time is defined as $\tau_{*}=\inf \left\{t \geq 0: B_{t} \leq g\left(S_{t}\right)\right\}$ with $g(x)=x-\frac{H^{\prime}(x)}{2 c}$. Let us determine the differential equation satisfied by $g$. Note that $H$ is by definition nondecreasing, so we have $H^{\prime}(x)=\sqrt{4 c} \sqrt{(H(x)-\phi(x))}$. For suitable $\phi$, this is a differentiable function and differentiating it we obtain

$$
H^{\prime \prime}(x)=\frac{2 c\left(H^{\prime}(x)-\phi^{\prime}(x)\right)}{\sqrt{4 c(H(x)-\phi(x))}}=\frac{2 c\left(H^{\prime}(x)-\phi^{\prime}(x)\right)}{H^{\prime}(x)} .
$$

Therefore

$$
g^{\prime}(x)=1-\frac{H^{\prime \prime}(x)}{2 c}=\frac{\phi^{\prime}(x)}{H^{\prime}(x)}=\frac{\phi^{\prime}(x)}{2 c(x-g(x))} .
$$

We recognize immediately the equation (1.3) only there $\phi^{\prime}(s)=1$ and $c$ was a function and not a constant. This motivated our investigation of the generalization of problem 1.1 given in 2.4 which is solved in Theorems 5 - 7 in Section 4

Consider now the converse problem. That is, given a centered probability measure $\mu$ describe all pairs of functions $(\phi, c)$ such that the optimal stopping time $\tau_{*}$, which solves (1.1) exists and embeds $\mu$, that is $B_{\tau_{\mu}} \sim \mu$.

This was called the "optimal Skorokhod embedding problem" (term introduced by Peskir in [14]), as we not only specify a method to obtain an embedding for $\mu$ but also construct an optimal stopping problem, of which this embedding is a solution.

Again, let us consider two special cases. First, let $\phi(x)=x$. From the Theorem 1 we know that $\tau_{*}$ is the Azéma-Yor stopping time and the function $g_{*}$ is just the inverse of barycentre function of some measure $\mu$. The problem therefore consists in identifying the dependence between $\mu$ and $c$. Suppose for simplicity that $\mu$ has a strictly positive density $f$ and that it satisfies $L \log L$-integrability condition.

Theorem 3 (Peskir 14]). In the setup above there exists a unique function

$$
c(x)=\frac{1}{2} \frac{f(x)}{\bar{\mu}(x)}
$$

such that the optimal solution $\tau_{*}$ of 1.1) embeds $\mu$. This optimal solution is then the Azéma-Yor stopping time given by

$$
\begin{gathered}
\tau_{*}=\inf \left\{t \geq 0: S_{t} \geq \Psi_{\mu}\left(B_{t}\right)\right\}, \text { where } \\
\Psi_{\mu}(x)=\frac{1}{\mu([x, \infty))} \int_{[x, \infty)} y d \mu(y) .
\end{gathered}
$$

The function $c$ in (1.6) is recognized as a half of the hazard function. which plays an important role in some studies. Now let $c(x)=c$ be constant. Then we have

Theorem 4 (Meilijson 9). In the setup above, there exists a unique function $\phi$ defined through (1.4) with $H^{\prime}(x)=2 c\left(x-\Psi_{\mu}^{-1}(x)\right)$, where $\Psi_{\mu}$ is the barycentre function given in [1.8), such that the optimal solution $\tau_{*}$ of (2.4) embeds $\mu$. This optimal solution is then the Azéma-Yor stopping time given by 1.7). 
We will provide a general solution that identifies all the pairs $(\phi, c)$ in Proposition 8 in Section 5

\section{Notation}

In this small section we fix the notation that, unless stated otherwise, is used in the rest of the paper.

$X=\left(X_{t}\right)_{t \geq 0}$ denotes a real-valued, time-homogenous, regular diffusion associated with the infinitesimal generator

$$
\mathbb{L}_{X}=\delta(x) \frac{\partial}{\partial x}+\frac{\sigma^{2}(x)}{2} \frac{\partial^{2}}{\partial x^{2}},
$$

where the drift coefficient $x \rightarrow \delta(x)$ and the diffusion coefficient $x \rightarrow$ $\sigma(x)>0$ are continuous. We assume moreover that there exists a realvalued, standard Brownian motion $B=\left(B_{t}\right)_{t \geq 0}$, such that $X$ solves the stochastic differential equation

$$
d X_{t}=\delta\left(X_{t}\right) d t+\sigma\left(X_{t}\right) d B_{t},
$$

with $X_{0}=x_{0}$ under $\mathbb{P}_{x}:=\mathbb{P}$ for $x \in \mathbb{R}$. $\left(\mathcal{F}_{t}\right)_{t \geq 0}$ is the natural filtration of $X$. The scale function and the speed measure of $X$ are denoted respectively by $L$ and by $m$. The state space of $X$ is $\left(a_{X}, b_{X}\right)$, where $a_{X}<b_{X}$ can be finite or infinite. The interval $\left(a_{X}, b_{X}\right)$ might be also closed from one, or both, sides under some additional conditions, as will be discussed later. We recall, that for Brownian motion $L(x)=x$ and $m(d x)=2 d x$.

The one-sided supremum process associated with $X$ is

$$
S_{t}=\left(\sup _{0 \leq r \leq t} X_{r}\right) \vee s
$$

started at $s \geq x$ under $\mathbb{P}_{x, s}:=\mathbb{P}$. When several supremum processes will appear for different diffusions, we will add appropriate superscripts, thus writing $S_{t}^{X}$. The first hitting times for $S$ are noted $T_{a}=\inf \left\{t \geq 0: S_{t} \geq\right.$ $a\}$.

We make the following assumptions on the function $\phi$ :

- $\phi:\left(a_{X}, b_{X}\right) \rightarrow \mathbb{R}$ is non-decreasing, right-continuous and its points of discontinuity are isolated;

- $\phi^{\prime}$, taken right-continuous, is well defined and its points of discontinuity are isolated and countable;

- there exists $r_{\phi}$, such that on $\left[r_{\phi}-1,+\infty\right), \phi \in C^{1}$ and $\phi^{\prime}>0$;

The last assumption has technical signification and is there to allow for a convenient identification of a particular solution of a differential equation. It will be clear form our proofs of main theorems, we hope, that one could take many other conditions, which would yield different descriptions.

We denote $D_{\phi}$, the set of points of discontinuity of $\phi$. It is bounded from above by $r_{\phi}$ and can write $D_{\phi}=\left\{d_{0}, d_{1}, \ldots\right\}$ where $d_{i} \geq d_{i+1}$. Similarly, the set of points of discontinuity of $\phi^{\prime}$ is also bounded from above by $r_{\phi}$ and we can write $D_{\phi^{\prime}}=D_{\phi} \cup\left\{f_{0}, f_{1}, \ldots\right\}$, where $f_{i} \geq f_{i+1}$. 
It will be convenient to work sometimes with a different function $\tilde{\phi}$, which is of class $C^{1}$ with $\tilde{\phi}^{\prime}>0$, and satisfies: $\tilde{\phi} \leq \phi, \tilde{\phi}_{\left.\right|_{\left[r_{\phi}, \infty\right)}}=\phi_{\left.\right|_{\left[r_{\phi}, \infty\right)}}$. No reasoning will depend on a particular choice of $\tilde{\phi}$, so we do not present a specific construction.

The function $c:[0,+\infty) \rightarrow[0,+\infty)$ has a countable number of discontinuities. In his work Peskir 13 supposed $c>0$, but we will see (cf. Remark 5] that allowing $c$ to be zero on some intervals can be of great use. In some parts of the work we will make additional assumptions on the regularity of $c$, yet in others we will allow it to take the value $+\infty$.

We consider the following optimal stopping problem:

$$
V_{*}(x, s)=\sup _{\tau} \mathbb{E}_{x, s}\left(\phi\left(S_{\tau}\right)-\int_{0}^{\tau} c\left(X_{t}\right) d t\right),
$$

where $\tau$ is a $\mathcal{F}_{t}$-stopping time, which satisfies

$$
\mathbb{E}_{x, s}\left(\int_{0}^{\tau} c\left(X_{t}\right) d t\right)<\infty
$$

Through a solution of "the optimal stopping problem (2.4)" we understand a stopping time which yields $V_{*}$ and satisfies the condition (2.5). Such solution is denoted $\tau_{*}$, i.e. $V_{*}(x, s)=\mathbb{E}_{x, s}\left(\phi\left(S_{\tau_{*}}\right)-\int_{0}^{\tau_{*}} c\left(X_{t}\right) d t\right)$. Note that of course $V_{*}$ and $\tau_{*}$ depend on $\phi$ and $c$ but it will be obvious from the context if we discuss general situation of arbitrary $(\phi, c)$ or some special cases.

\section{Some remarks on the problem}

In the next section we will present a complete solution of the optimal stopping problem (2.4). However, before we do it, we want to give some relatively simple observations, which help to understand better the nature of the problem. Most of them become nearly evident once a person is acquainted with the problem, yet we think it is worthwhile to gather them here. As a matter of fact, they will help us a great deal, both in formulating and in proving, the main results of this paper.

The general construction of the optimal stopping problem under consideration is such, that we get rewarded according to the record-high value so far, and get punished all the time, proportionally to the time elapsed and depending on the path of our process. This means basicly that the situation when the diffusion increases is favorable and what is potentially dangerous, as it might be "too costly", are big excursions below the supremum, that is negative excursions of the process $\left(X_{t}-S_{t}\right)$. The first observation is then that we should not stop in the support of $d S_{t}$.

Remark 1 (Proposition 2.1 [13]). If $\phi^{\prime}>0$ and $\tau$ yields the solution to the problem (2.4), then $X_{\tau} \neq S_{\tau}$ a.s., that is the process $\left(X_{t}, S_{t}\right)$ can not be optimally stopped on the diagonal of $\mathbb{R}^{2}$.

Let us investigate in more detail the nature of the optimal stopping time. If we follow the process $X_{t}$, as noted above, we gain when it increases. In contrast, when it decreases we only get punished all the time. 
This means that at some point it gets too costly to continue a given negative excursion of $\left(X_{t}-S_{t}\right)$. Exactly when it becomes too costly to continue the excursion depends only on three factors: the diffusion characteristics, the functions $\phi$, and $c$, and on the value $S_{t}$ of the supremum so far. Indeed, the choice of the point in an obvious manner depends on what we expect to earn, if we get back to $S_{t}$, which in turn is a function of the diffusion characteristics and the functions $\phi$, and $c$. Note however, that through the Markov property, it doesn't depend on the way we will arrive at $S_{t}$. Similarly, thanks to the Markov property, the way the excursion of $(X-S)$ straddling time $t$ develops, doesn't affect the choice of the stopping value.

Remark 2. The solution to the optimal stopping problem 2.4), if it exists, is given by a stopping time of the following form $\tau=\inf \{t \geq 0$ : $\left.X_{t} \leq g_{*}\left(S_{t}\right)\right\}$, where the function $g_{*}$ depends on the characteristics of $X$ and the functions $\phi$, and $c$.

Now that we have a feeling of how the stopping time looks like, we will try to learn more about the function $g_{*}$. Consider the function $V(x, s)$ given by (2.4). Since $V(x, s)$ corresponds to the optimal stopping of the process $\left(X_{t}, S_{t}\right)$ started at $(x, s)$, the values of $\phi(u)$ for $u<s$ never intervene. Furthermore, if the process reaches some value $s_{1}>s$ then, thanks to the strong Markov property, we just face the problem $V_{\sim}\left(s_{1}, s_{1}\right)$. We could then replace the function $\phi$ with some other function $\tilde{\phi}$ which coincides with $\phi$ on $\left[s_{1}, \infty\right)$ and this would not affect the stopping rule, that is it would not affect values of the function $g_{*}$ on the interval $\left[s_{1}, \infty\right)$. Furthermore, the finiteness of $V$ is not affected by any change of $\phi$ away from infinity.

Remark 3. Take two functions $\phi_{1}$ and $\phi_{2}$ which coincide on some halfline: $\phi_{1}(s)=\phi_{2}(s)$ for all $s \geq r$. The optimal stopping problem 2.4) for $\phi_{1}$ has finite payoff if and only if the optimal stopping problem (2.4) for $\phi_{2}$ has finite payoff. Suppose this the case and denote respectively $\tau_{1}=\inf \left\{t \geq 0: X_{t} \leq g_{*}^{1}\left(S_{t}\right)\right\}$ and $\tau_{2}=\inf \left\{t \geq 0: X_{t} \leq g_{*}^{2}\left(S_{t}\right)\right\}$ the stopping times, which yield this payoffs. Then $g_{*}^{1}(s)=g_{*}^{2}(s)$ for all $s \geq r$.

We turn now to examine in more depth the relation between $\phi$ and $g_{*}$. Consider an interval of constancy of $\phi$. Suppose that $\phi(\alpha)=\phi(\beta)$ for some $\alpha<\beta$ and $\phi$ increases on right of $\beta$ (or has a jump). Then starting the process at $x \in[\alpha, \beta)$ we face always the same problem: we get punished all the time, but get rewarded only if we attain the level $\beta$. Due to the Markov character of the diffusion, it is clear that the optimal stopping time is then just an exit time of some interval $[\gamma, \beta]$ and $\gamma$ is then precisely the value of $g_{*}$ on $(\alpha, \beta)$. We conclude therefore, that

Remark 4. The functions $\phi$ and $g_{*}$, which induces the optimal stopping time, have the same intervals of constancy. In other words $g_{\left.*\right|_{(\alpha, \beta)} ^{\prime}} \equiv 0 \Leftrightarrow$ $\phi_{(\alpha, \beta)}^{\prime} \equiv 0$.

It is important to realize that if an interval of constancy of $\phi$ is long and the function doesn't grow fast enough afterwards, it may be optimal to stop immediately when coming to such a level of constancy, or even before. Thus the function $g_{*}$ may stay in part under and in part above the diagonal in $\mathbb{R}^{2}$. 
Analysis of jumps of $\phi$ and $g_{*}$ is more involved. When $\phi$ is continuous, since the diffusion itself is continuous too, it's not hard to believe that $g_{*}$ should be continuous as well. It is maybe a little more tricky to convince yourself that if $\phi$ jumps, then $g_{*}$ has a jump too. We will try to argue this here, and moreover we will determine the height of the jump, following the reasoning of Meilijson 9] developed in the proof of Corollary 1. Suppose that $\phi$ has a jump in $s_{0}: \phi\left(s_{0}\right)-\phi\left(s_{0}-\right)=j>0$. For simplicity, suppose also that $\phi$ is constant on some interval before the jump: $\phi\left(s_{0}-\epsilon\right)=$ $\phi\left(s_{0}-\right)$. Then, as noted above, starting the process at some $x \in\left[s_{0}-\epsilon, s_{0}\right)$ we wait till the first exit time of the interval $\left[g_{*}(x), s_{0}\right]$. If we exit at the bottom we stop, and if we exit at the top, we have a new, independent diffusion starting, for which the expected payoff is just $V\left(s_{0}, s_{0}\right)$. Denote the exit time of an interval $[a, b]$, for a process starting at $y \in[a, b]$, through $\rho_{a, b}^{y}$, and the first hitting time of a level $a$, starting at $y$, by $\rho_{a}^{y}$. We can then write the payoff $V(x, x)$ as

$$
\begin{aligned}
V(x, x)= & \mathbb{E}\left[V\left(s_{0}, s_{0}\right) \mathbf{1}_{\left\{\rho_{g_{*}(x), s_{0}}^{x}=\rho_{s_{0}}^{x}\right\}}+\phi(x) \mathbf{1}_{\left\{\rho_{g_{*}(x), s_{0}}^{x}=\rho_{g_{*}(x)}^{x}\right\}}\right. \\
& \left.-\int_{0}^{\rho_{g_{*}(x), s_{0}}^{x}} c\left(X_{u}\right) d u\right] .
\end{aligned}
$$

We know, by Remark 3 that the value $g_{*}\left(s_{0}\right)$ is uniquely determined by the diffusion characteristics, the cost function $c$, and values of the function $\phi$ on $\left[s_{0}, \infty\right)$. Replace in the above display $g_{*}(x)$ by $a$ and denote this quantity $V^{a}(x, x)$. Then $V(x, x)=\sup _{a<s_{0}} V^{a}(x, x)$. This determines the value of $a_{*}^{x}=g_{*}(x)$ and therefore the height of the jump of $g_{*}$ at $s_{0}$. We will now show that the value of $a_{*}^{x}$ does not depend on $x$ (which is also clear by Remark 4) and give an equation which describes it.

Actually the equation for $a_{*}^{x}$ is obvious: $a_{*}^{x}$ is the unique real, such that $\left.\frac{\partial V^{a}(x, x)}{\partial a}\right|_{a=a_{*}^{x}}=0$. This can be rewritten in the following equivalent manner, using the fact that $\mathbb{P}\left(\rho_{a, b}^{x}=\rho_{b}^{x}\right)=(L(x)-L(a)) /(L(b)-L(a))$ :

$L^{\prime}\left(a_{*}^{x}\right) \cdot \frac{L\left(s_{0}\right)-L(x)}{\left(L\left(s_{0}\right)-L\left(a_{*}^{x}\right)\right)^{2}}\left(\phi(x)-V\left(s_{0}, s_{0}\right)\right)=\frac{\partial}{\partial a} \mathbb{E}\left[\int_{0}^{\rho_{a_{*}^{x}, s_{0}}^{x^{x}}} c\left(X_{u}\right) d u\right]$.

We just have to show that actually $a_{*}^{x}$ does not depend on $x$. To this end let $a<y<x<s_{0}$ and write

$$
\begin{aligned}
\mathbb{E}\left[\int_{0}^{\rho_{a, s_{0}}^{x}} c\left(X_{u}\right) d u\right] & =\mathbb{E}\left[\int_{0}^{\rho_{s_{0}}^{x}} c\left(X_{u}\right) d u \cdot \mathbf{1}_{\rho_{y, s_{0}}^{x}}=\rho_{s_{0}}^{x}\right. \\
& \left.+\int_{0}^{\rho_{y}^{x}} c\left(X_{u}\right) d u \cdot \mathbf{1}_{\rho_{y, s_{0}}^{x}=\rho_{y}^{x}}\right] \\
& +\frac{L(s)-L(x)}{L(s)-L(y)} \mathbb{E}\left[\int_{0}^{\rho_{a, s_{0}}^{y}} c\left(X_{u}\right) d u\right]
\end{aligned}
$$

where we used the strong Markov property at time $\rho_{y}^{x}$. The first two terms on the right-hand side do not depend on $a$, and differentiating the above equation with respect to $a$ yields an identity which proves that $a_{*}^{x}=a_{*}^{y}=: a_{*}$ for $x, y \in\left[s_{0}-\epsilon, s_{0}\right)$, since $\phi$ was supposed constant on this interval. It is also clear that $a_{*}$ depends only on the characteristics of $X$, 
the cost function $c$ and the payoff value $V\left(s_{0}, s_{0}\right)$. Since the diffusion is fixed, we write $a_{*}=a_{*}\left(c, V\left(s_{0}, s_{0}\right)\right)$.

So far we only analyzed the dependence between $g_{*}$ and $\phi$, and it's time to investigate the rôle of the cost function $c$. Suppose that the function $c$ disappears on some interval, $c_{\left.\right|_{(\alpha, \beta)}} \equiv 0$. Thus wandering away from the supremum in the interval $(\alpha, \beta)$ doesn't cost us anything - there is no reason therefore to stop while in this interval. This implies that $(\alpha, \beta)$ is not in the range of $g_{*}$.

Remark 5. If $c_{\left.\right|_{(\alpha, \beta)}} \equiv 0$ end $\tau_{*}$ yields the optimal payoff to the problem 2.4), then $(\alpha, \beta) \cap g_{*}(\mathbb{R})=\emptyset$ and $X_{\tau_{*}} \notin(\alpha, \beta)$ a.s.

In a sense then, intervals of disappearance of $c$ have the same impact on the function $g_{*}$ as jumps of $\phi$. Their impact is however much easier to describe. We could predict that jumps of $c$ would provoke a similar behavior of $g_{*}$ as intervals of constancy of $\phi$. This however proves to be untrue. It stems from the fact that the values of $c$ are averaged through integrating them. Thus, jumps of $c$ will only produce discontinuities of $g_{*}^{\prime}$. We will come back to this matter in Theorem [6

\section{Maximality principle revisited}

In this section we describe the main result of our paper, namely the complete solution to the optimal stopping problem (2.4). The solution is described in a sequence of three theorems with increasing generality of the form of the function $\phi$. In the first theorem we suppose that $\phi$ is of class $C^{1}$ and strictly increasing. Our theorem is basicly a re-writing of the Theorem 3.1 found in Peskir [13]. Theorem [6] treats the case of a continuous function $\phi$, and is obtained form the previous one through an approximation procedure. The last theorem is less explicit in a sense, as the treatment of jumps of $\phi$ is harder. Our approach follows rather the work of Meilijson 9 .

Theorem 5 (Peskir). Let $\phi \in C^{1}$ with $\phi^{\prime}>0$, and $c>0$ be continuous. The problem 2.4), has an optimal solution with finite payoff if and only if there exists a maximal solution $g_{*}$ of

$$
g^{\prime}(s)=\frac{\phi^{\prime}(s) \sigma^{2}(g(s)) L^{\prime}(g(s))}{2 c(g(s))(L(s)-L(g(s)))}, \quad a_{X}<s<b_{X},
$$

which stays strictly below the diagonal in $\left(a_{X}, b_{X}\right)^{2}$ (i.e. $g_{*}(s)<s$ for $\left.a_{X}<s<b_{X}\right)$. More precisely, let $s_{*}$ such that $g_{*}\left(s_{*}\right)=a_{X}$. Then $g_{*}$ satisfies 4.1) on $\left(s_{*}, b_{X}\right)$ and it is maximal such function (where the functions are compared on the interval, where both of them are superior to $\left.a_{X}\right)$, which stays below the diagonal.

In this case the payoff is given by

$$
V_{*}(x, s)=\phi(s)+\int_{x \wedge g_{*}(s)}^{x}(L(x)-L(u)) c(u) m(d u),
$$

The stopping time $\tau_{*}=\inf \left\{t \geq 0: X_{t} \leq g_{*}\left(S_{t}\right)\right\}$ is then optimal whenever it satisfies (2.5), otherwise it is "approximately" optimal ${ }^{1}$.

\footnotetext{
${ }^{1}$ In the limit sense as in Peskir [13].
} 
Furthermore if there exists a solution $\rho$ of the optimal stopping problem 2.4) then $\mathbb{P}_{x, s}\left(\tau_{*} \leq \rho\right)=1$ and $\tau_{*}$ satisfies [2.5).

If there is no maximal solution of 4.1), which stays strictly below the diagonal in $\mathbb{R}^{2}$, then $V_{*}(x, s)=\infty$ for all $x \leq s$.

The last property for $\tau_{*}$ says it's just pointwise the smallest solution. It is a general property of the Azéma-Yor stopping time and we will not repeat it below, we refer to Obłój [10], chapter 8, for details. Note that for a general $\phi$, we defined in Section 2 the function $\tilde{\phi}$, which coincides with $\phi$ on the interval $\left[r_{\phi}, \infty\right)$ and is of class $C^{1}$. In particular, we can apply the above theorem to the optimal stopping problem (2.4) with $\phi$ replaced by $\tilde{\phi}$. We denote $\tilde{g}_{*}$ the function which gives the optimal stopping time $\tilde{\tau}=\inf \left\{t \geq 0: X_{t} \leq \tilde{g}_{*}\left(S_{t}\right)\right\}$, which solves this problem.

Theorem 6. Let $\phi$ be as described in Section 2 but continuous, and $c>0$ be continuous. Then the optimal stopping problem (2.4) has a finite payoff if and only if the optimal stopping problem with $\phi$ replaced by $\tilde{\phi}$ has a finite payoff. In this case there exists a continuous function $g_{*}$, which satisfies the differential equation (4.1) on the interior of the set $\mathbb{R} \backslash D_{\phi^{\prime}}$, and coincides with $\tilde{g}_{*}$ on the interval $\left[r_{\phi}, \infty\right)$. The payoff is given by the formula 4.2) and is obtained for the stopping time $\tau=\inf \left\{t \geq 0: X_{t} \leq\right.$ $\left.g_{*}\left(S_{t}\right)\right\}$, if it satisfies 2.5). Otherwise this stopping time is approximately optimal.

It is important to note, that the function $g_{*}$ may not satisfy anymore $g_{*}<I d$, since if the constancy intervals of $\phi$ are too long it might be optimal to stop immediately (see also discussion around Remark 4).

The formulation for the case of $\phi$ with discontinuities is somewhat more technical, as we have to define $g_{*}$ through an iteration procedure.

Theorem 7. Let $\phi$ be as described in Section 2 and $c>0$ be continuous. The optimal stopping problem [2.4) has finite payoff if and only if the optimal stopping problem with $\phi$ replaced by $\tilde{\phi}$ has a finite payoff. In this case there exists a function $g_{*}$, continuous on the set $\mathbb{R} \backslash D_{\phi}$, which satisfies the following:

- $g_{*}(s)=\tilde{g}_{*}(s)$ for all $s \in\left[r_{\phi}, \infty\right)$;

- $g_{*}$ is continuous on the interior of the set $\mathbb{R} \backslash D_{\phi}$ and differentiable on the interior of the set $\mathbb{R} \backslash D_{\phi^{\prime}}$, where it satisfies the equation [4.1];

- for all $s \in D_{\phi}, a_{*}=g_{*}(s-)$ satisfies the equation [3.2) with $s_{0}=s$.

The payoff is described via 4.2).

It is important to note, that even though the optimal payoff $V$ appears in the equation (3.2) the above construction of $g_{*}$ is feasible. Recall, that the jumps points were denoted $\ldots<d_{1}<d_{0}$. When determining the value of $g_{*}\left(d_{i}-\right)$ we already know the values of $g_{*}$ on $\left[d_{1}, \infty\right)$ and through the formula (4.2) also the payoff $V(x, s)$ for $d_{i} \leq x \leq s$.

Proofs. Theorem 5 Step 1. We start with the case when the state space of the diffusion $X$ is the whole real line, $\left(a_{X}, b_{X}\right)=\mathbb{R}$, and the function $\phi$ is strictly increasing and of class $C^{1}$, and its image is the real line $\phi: \mathbb{R} \stackrel{\text { on }}{\rightarrow} \mathbb{R}$. Let $Y_{t}=\phi\left(X_{t}\right)$. It is a diffusion with the scale function 
$L^{Y}(s)=L\left(\phi^{-1}(s)\right)$, the drift coefficient $\delta^{Y}(y)=\phi^{\prime}\left(\phi^{-1}(y)\right) \delta\left(\phi^{-1}(y)\right)$, the diffusion coefficient $\sigma^{Y}(y)=\phi^{\prime}\left(\phi^{-1}(y)\right) \sigma\left(\phi^{-1}(y)\right)$ and the speed measure $m^{Y}(d y)=\frac{2 d y}{L^{\prime}\left(\phi^{-1}(y)\right) \phi^{\prime}\left(\phi^{-1}(y) \sigma^{2}\left(\phi^{-1}(y)\right)\right.}$. The state space of $Y$ is the whole real line. We can rewrite the optimal stopping problem for $X$ in terms of $Y$ (where we add superscripts $X$ and $Y$ to denote the quantities corresponding to those two processes):

$$
\begin{aligned}
V_{*}^{X}(x, s) & =\sup _{\tau} \mathbb{E}_{x, s}\left(\phi\left(S_{\tau}^{X}\right)-\int_{0}^{\tau} c\left(X_{t}\right) d t\right) \\
& =\sup _{\tau} \mathbb{E}_{x, s}\left(S_{\tau}^{Y}-\int_{0}^{\tau} c\left(\phi^{-1}\left(Y_{t}\right)\right) d t\right) \\
& =V_{*}^{Y}(\phi(x), \phi(s)) \text { under } \tilde{\phi}=I d \text { and } \tilde{c}=c \circ \phi^{-1} .
\end{aligned}
$$

We see therefore that the optimal solutions for $X$ coincide with the optimal solution for $Y$ for a different problem. We can apply Theorem 3.1 in Peskir 13 for this new problem for $Y$ to obtain that the optimal solution is given by

$$
\begin{aligned}
\tau_{*} & =\inf \left\{t \geq 0: Y_{t} \leq g_{*}^{Y}\left(S_{t}^{Y}\right)\right\} \\
& =\inf \left\{t \geq 0: X_{t} \leq \phi^{-1}\left(g_{*}^{Y}\left(\phi\left(S_{t}^{X}\right)\right)\right)\right\},
\end{aligned}
$$

which yields $g_{*}^{X}(s)=\phi^{-1}\left(g_{*}^{Y}(\phi(s))\right)$. Note therefore that the existence of $g_{*}^{X}$ is equivalent to the existence of $g_{*}^{Y}$. We will now show that $g_{*}^{X}$ satisfies the equation (4.1). We will use the following simple observations: $\phi^{-1^{\prime}}(\phi(s)) \phi^{\prime}(s)=1$ and $\frac{1}{\phi^{-1^{\prime}}\left(g_{*}^{Y}(\phi(s))\right)}=\phi^{\prime}\left(g^{X}(s)\right)$ and the fact that $g_{*}^{Y}$ satisfies

$$
\begin{aligned}
g_{*}^{Y^{\prime}}(s)= & \frac{\phi^{\prime}\left(\phi^{-1}\left(g_{*}^{Y}(s)\right)\right)^{2} \phi^{-1^{\prime}}\left(g_{*}^{Y}(s)\right) \sigma^{2}\left(\phi^{-1}\left(g_{*}^{Y}(s)\right)\right) L^{\prime}\left(\phi^{-1}\left(g_{*}^{Y}(s)\right)\right)}{2 c\left(\phi^{-1}\left(g_{*}^{Y}(s)\right)\right)\left(L\left(\phi^{-1}(s)\right)-L\left(\phi^{-1}\left(g_{*}^{Y}(s)\right)\right)\right)} \\
g_{*}^{X^{\prime}}(s) & =\phi^{-1^{\prime}}\left(g_{*}^{Y}(\phi(s))\right) \cdot g_{*}^{Y^{\prime}}(\phi(s)) \cdot \phi^{\prime}(s) \\
& =\frac{\phi^{-1^{\prime}}\left(g_{*}^{Y}(\phi(s))\right)^{2}\left(\phi^{\prime}\left(g_{*}^{X}(s)\right)\right)^{2} \sigma^{2}\left(g_{*}^{X}(s)\right) L^{\prime}\left(g_{*}^{X}(s)\right) \phi^{\prime}(s)}{2 c\left(g_{*}^{X}(s)\right)\left(L(s)-L\left(g_{*}^{X}(s)\right)\right)} \\
& =\frac{\phi^{\prime}(s) \sigma^{2}\left(g_{*}^{X}(s)\right) L^{\prime}\left(g_{*}^{X}(s)\right)}{2 c\left(g_{*}^{X}(s)\right)\left(L(s)-L\left(g_{*}^{X}(s)\right)\right)},
\end{aligned}
$$

in which we recognize 4.1). Note that $\left(g_{*}^{X}(s)<s\right.$ for $\left.s \in \mathbb{R}\right) \Leftrightarrow\left(g_{*}^{Y}(s)<\right.$ $s$ for $s \in \mathbb{R})$ as $\left(g_{*}^{X}(s)<s\right) \Leftrightarrow\left(g_{*}^{Y}(s)<s\right)$ and $\phi$ is a one to one map from $\mathbb{R}$ to $\mathbb{R}$. This yields the description of $g_{*}^{X}$ as the maximal solution, which stays strictly below the diagonal in $\mathbb{R}^{2}$.

The expression for payoff follows also from Peskir [13] by a change of variables.

Step 2. In this step we extend the previous results to the case, when the diffusion $X$ or the function $\phi$ are not unbounded. First, observe that the results of Peskir work just as well for a diffusion with an arbitrary 
state space $\left(a_{X}, b_{X}\right),-\infty \leq a_{X}<b_{X} \leq \infty$. Indeed, if we take $\phi=I d$, the solution is given by the maximal solution of the equation (4.1) for $s \in\left(a_{X}, b_{X}\right)$ and which stays strictly below the diagonal in $\left(a_{X}, b_{X}\right)^{2}$. Formally, we could repeat the proof in of Theorem 3.1 in 13, in about the same manner that Peskir treats non-negative diffusions in Section 3.11 in the same article. Moreover, in this section, Peskir also discusses the boundary behavior, to which we will come below (notice that so far we considered unattainable boundaries, see [8, pages 226-236).

We conclude therefore that the assumption on the state space of the diffusion, as well as on the range of $\phi$, were superficial. We can just as well have $\phi: \mathbb{R} \rightarrow(a, b)$, we need to keep however $\phi^{\prime}>0$. The equation (4.5) is valid in $(a, b)$ then, but the original one (4.1) is valid in $\left(a_{X}, b_{X}\right)$ as asserted, due to the fact that $g_{*}^{X}=\phi^{-1} \circ g_{*}^{Y} \circ \phi$. The assumption, that $\phi$ is strictly increasing is needed for the new process to be a diffusion. We now turn to more general cases, which will be treated by approximation.

Theorem [6. We extend the previous results to functions $\phi$, which are just non-decreasing and continuous. This is done through an approximation procedure. That the payoff for the optimal stopping problem for $\phi$ is finite if and only if it is also finite for $\tilde{\phi}$ follows immediately from Remark 3

Let $\phi_{n}$ be an increasing sequence of strictly increasing $C^{1}$-functions, converging to $\phi$, such that $\phi_{n}^{\prime} \rightarrow \phi^{\prime}$. Note $V_{n}$ the payoff given in (2.4) for function $\phi_{n}$, under the condition (2.5). Obviously $V_{n} \leq V_{n+1} \leq V$. In particular the payoff $V$, associated with $\phi$, can be finite only if all $V_{n}$ are finite. Suppose this is the case. We know, by Theorem 5 proved above, that $V_{n}$ are given by a sequence of stopping times $\tau_{*}^{n}, \tau_{*}^{n}=\inf \{t \geq 0$ : $\left.X_{t} \leq g_{*}^{n}\left(S_{t}\right)\right\}$, where $g_{*}^{n}$ are defined as the maximal solutions of (4.1), (with $\phi$ replaced by $\phi_{n}$ ), which stay below the diagonal. It is easy to see that $\tau_{*}^{n} \leq \tau_{*}^{n+1}$ and therefore $g_{*}^{n} \geq g_{*}^{n+1}$. This is a direct consequence of the fact that in the problem (2.4) the reward $\left(\phi_{n}\left(S_{\tau}\right)\right)$ increases and the punishment $\left(\int_{0}^{\tau} c\left(X_{s}\right) d s\right)$ stays the same. It can also be seen from the differential equation (4.1) itself. We have therefore $\tau_{*}^{n} \nearrow \tau_{*}$ a.s., for some stopping time $\tau_{*}$, possibly infinite. Moreover, $\tau_{*}=\inf \{t \geq 0$ : $\left.X_{t} \leq g_{*}\left(S_{t}\right)\right\}$, where $g_{*}^{n} \searrow g_{*}$. Note that the limit is finite and satisfies $g_{*}(s)<s$ (which show that $\tau_{*}<\infty$ a.s.). Furthermore, we can pass to the limit in the differential equations describing $g_{*}^{n}$ to see that $g_{*}$ satisfies 4.1). Note that this agrees with Remark 4 Suppose that the stopping time $\tau_{*}$ satisfies (2.5). We then see in a straightforward manner, that $\tau_{*}$ solves the optimal stopping problem for $\phi$. It suffices to write:

$$
\begin{aligned}
\mathbb{E}_{x, s}\left(\phi\left(S_{\tau_{*}}\right)-\int_{0}^{\tau_{*}} c\left(X_{t}\right) d t\right) & \leq V_{*}(x, s) \\
& =\sup _{\tau} \mathbb{E}_{x, s}\left(\phi\left(S_{\tau}\right)-\int_{0}^{\tau} c\left(X_{t}\right) d t\right) \\
& \leq \lim _{n \rightarrow \infty} \sup _{\tau} \mathbb{E}_{x, s}\left(\phi_{n}\left(S_{\tau}\right)-\int_{0}^{\tau} c\left(X_{t}\right) d t\right) \\
& =\lim _{n \rightarrow \infty} \mathbb{E}_{x, s}\left(\phi_{n}\left(S_{\tau_{*}^{n}}\right)-\int_{0}^{\tau_{*}^{n}} c\left(X_{t}\right) d t\right) \\
& =\mathbb{E}_{x, s}\left(\phi\left(S_{\tau_{*}}\right)-\int_{0}^{\tau_{*}} c\left(X_{t}\right) d t\right)
\end{aligned}
$$


where passing to the limit in both cases is justified by the monotone convergence of $\phi_{n} \nearrow \phi$ and $\tau_{*}^{n} \nearrow \tau_{*}$ a.s.. The expression for payoff given in (4.2) follows also upon taking the limit.

If the stopping time $\tau_{*}$ fails to satisfy (2.5), we proceed as Peskir (cf. page 12, 13), to see that the expression (4.2) for payoff remains true (and we say $\tau^{*}$ is approximately optimal, as it is in fact a limit of stopping times satisfying (2.5).

Theorem 7 . All that has to be verified is the expression for $g_{*}\left(d_{i}-\right), i \geq$ 0 , where $D_{\phi}=\left\{d_{0}, d_{1}, \ldots\right\}$. Obviously, it is enough to discuss one jump, say $d_{0}$. The proposed value for $g\left(d_{0}-\right)$ is just the value we obtained at the end of Section 3 We had however an assumption that $\phi$ was constant on some interval $\left[d_{0}-\epsilon, d_{0}\right)$ and we need to argue that it can be omitted. This is done by approximating $\phi$. Define $\phi_{n}$ through $\phi_{n}(s)=\phi(s)$ for all $s \in \mathbb{R} \backslash\left[d_{0}-\frac{1}{n}, d_{0}\right)$ and $\phi_{n}$ constant and equal to $\phi\left(d_{0}-\right)$ on $\left[d_{0}-\frac{1}{n}, d_{0}\right)$. The theorem then applies to functions $\phi_{n}$, as by Remark 3$]$ the finiteness of the payoff for $\phi, \tilde{\phi}$ and $\phi_{n}$ are equivalent. Passing to the limit, as in the proof of Theorem [6]above, yields the result. Seemingly, taking a sequence of continuous functions $\tilde{\phi}_{n}$ increasing to $\phi$ and repeating the reasoning in (4.6) we see that the payoff equation (4.2) is still valid.

Note that $g_{*}\left(b_{X}\right)=b_{X}$. It is evident when the state space is $\left(a_{X}, b_{X}\right]$, since we have to stop upon achieving the upper bound for the supremum $S$, as continuation will only decrease the payoff. Generally, if $g_{*}\left(b_{X}\right)=$ $\beta<b_{X}$ (understood as limit if necessary), then as $S_{t} \rightarrow b_{X}$, the possible increase of payoff goes to zero but the cost till stopping doesn't.

The value of $g_{*}$ at $a_{X}$ depends on the boundary behavior of $X$ at $a_{X}$. Peskir [13] dealt with this subject in detail. He considered the case $a_{X}=0$, as it was motivated by applications, but this has no impact on the result. We state briefly the results and refer to his work for more details. When $a_{X}$ is a natural or exit boundary point, then $g_{*}\left(a_{X}\right)=\lim _{s \downarrow a_{X}} g_{*}(s)=a_{X}$. In contrast, when $a_{X}$ is an entrance or regular instantaneously reflecting boundary then $g_{*}\left(a_{X}\right)=\lim _{s \downarrow a_{X}} g_{*}(s)<a_{X}$.

\section{General optimal Skorokhod embedding problem}

In this section we consider the so-called optimal Skorokhod embedding problem. The general idea is to design, for a given measure $\mu$, such an optimal stopping problem with finite payoff, that the stopping time, which solves it, embeds $\mu$. We choose here to restrain ourselves to the classical setup of Brownian motion, $X=B$. Study for general diffusions is equally possible, only the formulae would be more complicated and lessintuitive. In principle however it is not harder, since we know very well how to rephrase the classical Azéma-Yor embedding for the setup of any real diffusion (Azéma-Yor [2, Cox and Hobson [3], see Obłój 10] for a complete description of the subject).

This problem was first introduced in the context of classification of contingent claims, where the measure $\mu$ has an interpretation in terms 
of risk associated with an option (see Peskir [16]). Peskir in his article on the optimal Skorokhod embedding 14 gave an explicit solution, but he assumed for simplicity that the measure $\mu$ has positive density on $\mathbb{R}$. One could think that this will generalize easily to arbitrary measures. Unfortunately this proves not to be true. In the setup when $\phi(x)=x$, it is not possible to solve the optimal embedding problem for a measure, which has an atom in the interior of its support. To see this, note that the presence of an atom in the interior of the support implies that the barycentre function $\Psi_{\mu}$ has a jump, which in turn means that the function $g_{*}=\Psi_{\mu}^{-1}$ has to be constant on some interval. However, the derivative of $g$ is given by $g^{\prime}(s)=[2 c(g(s))(s-g(s))]^{-1}$ which is strictly positive. In this sense, it is the presence of the function $\phi$ which proves fundamental to solve the problem. Meilijson 9], who had $c(x)=c$ but arbitrary $\phi$, solved this problem for any centered measure $\mu$ with finite variance, but his solution is not really explicit.We will see that actually to obtain a general explicit solution, both function $c$ and $\phi$ are needed, as the former allows to treat the regular (absolutely continuous) part of a measure, and the latter serves to obtain atoms.

We set ourselves two goals. Firstly, for a given measure $\mu$, we want to identify all the pairs $(\phi, c)$ such that the optimal stopping time $\tau_{*}$ which solves (2.4) embeds $\mu$ in $B, B_{\tau_{*}} \sim \mu$. We are interested in stopping times $\tau_{*}$ such that $\left(B_{t \wedge \tau_{*}}\right)$ is uniformly integrable. This limits the class of measures which are admissible to $\mu$ such that $\int_{\mathbb{R}}|x| d \mu(x)<\infty$ and $\int_{\mathbb{R}} x d \mu(x)=0$. Our second goal is to give a particular explicit description of some pair $(\phi, c)$.

Lest us start with the case of a measure $\mu$ with a positive density $f>0$ on $\mathbb{R}$. Then, we know that for $\tau_{*}=\inf \left\{t \geq 0: B_{t} \leq g_{*}\left(S_{t}\right)\right\}$ we have $B_{\tau_{*}} \sim \mu$ if and only if $g_{*}(s)=\Psi_{\mu}^{-1}(s)$, where $\Psi_{\mu}^{-1}$ denotes the inverse of $\Psi_{\mu}$. This is a direct consequence of the Azéma-Yor embedding [2] and the fact that $g$ is increasing with $g(s)<s$. Let us investigate the differential equation satisfied by $\Psi_{\mu}^{-1}$. Using the explicit formula (1.8), we obtain

$$
\left(\Psi_{\mu}^{-1}(s)\right)^{\prime}=\frac{\bar{\mu}\left(\Psi_{\mu}^{-1}(s)\right)}{\left(s-\Psi_{\mu}^{-1}(s)\right) f\left(\Psi_{\mu}^{-1}(s)\right)} .
$$

Comparing this with the differential equation for $g_{*}$ given by 4.1], we see that we need to have

$$
\frac{\phi^{\prime}(s)}{2 c\left(\Psi_{\mu}^{-1}(s)\right)}=\frac{\bar{\mu}\left(\Psi_{\mu}^{-1}(s)\right)}{f\left(\Psi_{\mu}^{-1}(s)\right)} .
$$

Equivalently, we have

$$
\frac{\phi^{\prime}\left(\Psi_{\mu}(u)\right)}{2 c(u)}=\frac{\bar{\mu}(u)}{f(u)}, \quad \text { for } u \in \mathbb{R} .
$$

Finally, we have to ensure that the payoff is finite. This is easy since we want $\mathbb{E} \phi\left(S_{\tau_{*}}\right)$ to be finite and we know that $S_{\tau_{*}}=\Psi_{\mu}\left(B_{\tau_{*}}\right)$, which implies that we need to have

$$
\int \phi\left(\Psi_{\mu}(x)\right) d \mu(x)<\infty
$$

We have thus obtained the following proposition: 
Proposition 8. Let $\mu$ be a probability measure on $\mathbb{R}$ with a strictly positive density $f$.Then the optimal stopping problem 2.4 has finite payoff and the stopping time $\tau_{*}$, which yields it embeds $\mu$ in $B$, i.e. $B_{\tau_{*}} \sim \mu$ if and only if $(\phi, c)$ satisfy (5.3) and (5.4) for all $s \geq 0$. The stopping time $\tau_{*}$ is then just the Azéma-Yor stopping time given by (1.7).

Note that, if we take $\phi(s)=s$ so that $\phi^{\prime}(s)=1$ we obtain a half of the hazard function for $c$, which is the result of Peskir, stated in Theorem 3 The integrability condition (5.4) then reads $\int \Psi_{\mu}(x) d \mu(x)<\infty$, which is known (see Azéma-Yor [1]) to be equivalent to the $L \log L$ integrability condition on $\mu$ : $\int_{1}^{\infty} x \log x d \mu(x)<\infty$.

Proof. In light of the reasoning the led to (5.3) all we have to comment on is the continuity of the function $c$. Theorems 517 were formulated for a continuous function $c>0$. Here, keeping the condition $c>0$, we drop the assumption on continuity. Still, this is not a problem. It suffices to take a sequence of functions $c_{n} \searrow c$ a.e. and proceed as in the proof of Theorem [6] around (4.6), to see that the function $g_{*}$, which yields the solution $\tau_{*}=\inf \left\{t \geq 0: B_{t} \leq g_{*}\left(S_{t}\right)\right\}$ to the optimal stopping problem (2.4), satisfies locally the equation (4.1) and is continuous. This in turn implies that it is indeed the inverse of the barycentre function associated with $\mu$.

Identifying all pairs $(\phi, c)$, which solve the optimal Skorokhod embedding problem for an arbitrary measure is harder. More precisely it's just not explicit any more. The reason is hidden in Theorem 7 - the description of jumps of $g_{*}$, which correspond to intervals not charged by the target measure, is done through an iteration procedure and is not explicit. This is exactly the reason why Meilijson was only able to prove the existence of the solution to the optimal Skorokhod embedding without giving any explicit formulae. In our approach we will use the duality between $\phi$ and $c$ to encode explicitly both the jumps and the regular part of the target measure. Still, we will not be able unfortunately to cover all probability measures.

Let $\mu$ be a centered probability measure $\int_{\mathbb{R}}|x| d \mu(x)<\infty, \int_{\mathbb{R}} x d \mu(x)=$ 0 and note $-\infty \leq a_{\mu}<b_{\mu} \leq+\infty$ respectively the lower, and the upper, bound of its support. Suppose $\mu$ is a sum of its regular and atomic parts: $\mu=\mu_{r}+\mu_{a}, d \mu_{r}(x)=f(x) d x$ and $\mu_{a}=\sum_{i \in \mathbb{Z}} p_{i} \delta_{j_{i}}$. In other words $f$ is the density of the absolutely continuous (with respect to the Lebesgue measure) part and $\cdots<j_{-1}<j_{0}<j_{1}<\ldots$ are the jump points of $\mu$, which are also the jump points of the barycentre function $\Psi_{\mu}$, so that $\Psi_{\mu}(\mathbb{R})=\mathbb{R}_{+} \backslash\left(\bigcup_{i \in \mathbb{Z}}\left(\Psi_{\mu}\left(j_{i}\right), \Psi_{\mu}\left(j_{i}+\right)\right]\right)$. We may note that $\Psi \mu\left(j_{i}+\right)=$ $\Psi_{\mu}\left(j_{i}\right)+\frac{p\left(\Psi_{\mu}\left(j_{i}\right)-j\right)}{\mu((j,+\infty))}$.

Theorem 9. In the above setup, in the case of $f>0$ or $f \geq 0$ but $\mu_{a}=0$, the optimal stopping problem 2.4) with

$$
c(x)=\left\{\begin{array}{ll}
\frac{f(x)}{2 \bar{\mu}(x)} & , \text { for } x \in\left[a_{\mu}, b_{\mu}\right] \\
+\infty & , \text { for } x \in \mathbb{R} \backslash\left[a_{\mu}, b_{\mu}\right]
\end{array} \quad \text { and } \quad \phi^{\prime}(x)=\mathbf{1}_{\Psi_{\mu}(\mathbb{R})}(x),\right.
$$

has finite payoff, which is realized by the Azéma-Yor stopping time $\tau_{*}=$ $\inf \left\{t \geq 0: S_{t} \geq \Psi_{\mu}\left(B_{t}\right)\right\}$. 
Note that the function $\phi$ is defined up to an additive constant, as adding a constant to $\phi$ doesn't affect the solution $\tau_{*}$ of (2.4).

Proof. The problem arising form discontinuity of $c$ is treated exactly as in the proof of Proposition 8 above. In the case of $f>0$ on $\mathbb{R}$ the above theorem follows immediately from Theorem $\mathbf{7}$ If $\mu$ has no atoms, then $\Psi_{\mu}(\mathbb{R})=\mathbb{R}_{+}$and so $\phi=I d$. From the differential equation (4.1) we can derive a differential equation for the inverse of $g_{*}$, from which is it clear that we can treat intervals where $c$ is zero by passing to the limit. So that also in the case of absolutely continuous measure but with a density which can be zero at some intervals our theorem is valid. Finally, the case of atoms with $\lim \sup _{n \rightarrow \infty} j_{n}=+\infty$ is also handeled upon approximating and taking the limit. Indeed this does not bother us here as we do not need anymore to describe the limiting solution through a differential equation, as was the case for Theorems [5- [7]

Unfortunately, our method does not work for an arbitrary measure $\mu$. If one tries to apply it for a purely atomic measure, he would end up with $\phi \equiv c \equiv 0$. In fact, when there is no absolutely continuous part we need to take $\phi$ discontinuous, as Meilijson does. We are not able to solve the problem explicitly then. We can not treat measures with singular non-atomic component.

\section{Important inequalities}

In the above section we saw how the solution to the optimal stopping problem yields a solution to the so-called optimal Skorokhod embedding problem. This actually motivated our research but is by no means a canonical application of the maximality principle. Probably the main and the most important applications are found among stopping inequalities. We will try to present some of them here. A specialist in the optimal stopping theory will find nothing new in this section and can probably skip it, yet we think it is useful to put it in this note, as it completes our study and allows us to give some references for further research. The main interest of the method presented here, is that the constants obtained are always optimal. Some of the inequalities below, as 6.5), are easy to obtain with 'some' constant. It was however the question of the optimal constant which stimulated researchers for a certain time.

Consider a continuous local martingale $X=\left(X_{t}: t \geq 0\right)$ and a stopping time $T$ in the natural filtration of $X, T<\infty$ a.s. We have then

$$
\mathbb{E} S_{T}^{X} \leq \sqrt{\mathbb{E} X_{T}^{2}}, \quad \text { where } S_{t}^{X}=\sup _{s \leq t} X_{s}
$$

and this is optimal. This simple inequality was first observed by Dubins and Schwarz [5]. It can be easily seen in the following manner (localizing and passing to the limit if necessary): $\mathbb{E} S_{T}=\mathbb{E}\left[S_{T}-X_{T}\right] \leq$ $\sqrt{\mathbb{E}\left[S_{T}-X_{T}\right]^{2}}=\sqrt{\mathbb{E}\left[X_{T}^{2}\right]}$, where we used the fact that $\left(S_{t}-X_{t}\right)^{2}-X_{t}^{2}$ is a local martingale (see Azéma and Yor [2]). This inequality is optimal as the equality is attained for $T=\inf \left\{t \geq 0: S_{t}^{X}-X_{t}=a\right\}$ for any $a>0$ $\left(X_{T}\right.$ has then shifted exponential distribution with parameter $\left.\frac{1}{a}\right)$. If we 
want to establish an analogous inequality for $|X|$, that is for a submartingale, some more care is needed. We propose to follow Peskir [12, 13] in order to obtain more general inequalities.

Consider the optimal stopping problem (2.4), with $X=|B|$ the absolute value of a Brownian motion, $\phi(s)=s^{p}$ and $c(x)=c x^{p-2}$, where $p>1$. We solve the problem applying Theorem [5] The differential equation (4.1) takes the form

$$
g^{\prime}(s)=\frac{p s^{p-1}}{2 c g(s)^{p-2}(s-g(s))},
$$

which is solved by $g(s)=\alpha s$, where $\alpha$ is the larger root of the equation $\alpha^{p-1}-\alpha^{p}=\frac{p}{2 c}$, which is seen to have solutions for $c \geq p^{p+1} / 2(p-1)^{(p-1)}$. Taking $c \searrow p^{p+1} / 2(p-1)^{(p-1)}$ yields

$$
\mathbb{E}\left(\max _{0 \leq t \leq \tau}\left|B_{\tau}\right|^{p}\right) \leq\left(\frac{p}{p-1}\right)^{p} \mathbb{E}\left|B_{\tau}\right|^{p},
$$

where $\tau$ is any stopping time in the natural filtration of $|B|$ such that $\mathbb{E} \tau^{p / 2}<\infty$. Note that to obtain the right-hand side of the above inequality we used the fact that $\mathbb{E}\left(\int_{0}^{\tau}\left|B_{t}\right|^{p-2}\right)=\frac{2}{p(p-1)} \mathbb{E}\left|B_{\tau}\right|^{p-1}$. More generally we could consider the optimal stopping problem (2.4) with $\phi(s)=s^{p}$ and $c(x)=c x^{q-1}$ for $0<p<q+1, q>0$. Optimizing upon $c$ Peskir [12] obtains

$$
\mathbb{E}\left(\max _{0 \leq t \leq \tau}\left|B_{\tau}\right|^{p}\right) \leq \gamma_{p, q}^{*} \mathbb{E}\left(\int_{0}^{\tau}\left|B_{t}\right|^{q-1} d t\right)^{p /(q-1)}
$$

for all stopping times $\tau$ of $|B|$. The optimal constant $\gamma_{p, q}^{*}$ is given implicitly as a solution of an equation. In the case $p=1$ it can be written explictly, $\gamma_{1, q}^{*}=(q(1+q) / 2)^{1 /(1+q)}(\Gamma(2+1 / q))^{q /(1+q)}$. This was obtained independently by Jacka 7 and Gilat 6 . In particular we find $\gamma_{1,1}^{*}=\sqrt{2}$ which is exactly the value found by Dubins and Schwarz [5]. If we consider the well-known bounds

$$
c_{p} \mathbb{E}\left(\int_{0}^{\tau}\left|B_{t}\right|^{p-2} d t\right) \leq \mathbb{E}\left(\max _{0 \leq t \leq \tau}\left|B_{\tau}\right|^{p}\right) \leq C_{p} \mathbb{E}\left(\int_{0}^{\tau}\left|B_{t}\right|^{p-2} d t\right),
$$

where $p>1$ and $c_{p}, C_{p}$ are some universal constants, we see that the inequality 6.4 complements them for $0<p \leq 1$, where 6.5 wouldn't have much sense. Also, as pointed out above, the method presented here allows to recover the optimal constants.

The inequalities given above are just a sample of applications of the method presented in this note. Peskir [13], for example, develops also a $L \log L$-type inequalities and some inequalities for Geometric Brownian motion. Although the method presented is very general it has of course its limits. For example it seems one could not recover optimal constants ${ }^{2}$ $a_{p}$ and $A_{p}$, obtained by Davis 4, such that for any stopping time $T$

$$
\begin{aligned}
& \mathbb{E}\left|B_{T}\right|^{p} \leq A_{p} \mathbb{E} T^{p / 2}, \text { for } 0<p<\infty, \text { and } \\
& a_{p} \mathbb{E} T^{p / 2} \leq \mathbb{E}\left|B_{t}\right|^{p}, \text { for } 1<p<\infty, \mathbb{E} T^{p / 2}<\infty .
\end{aligned}
$$

\footnotetext{
${ }^{2}$ These can be recovered using different methodology involving determinist time changing (see Pedersen and Peskir [11).
} 
The maximality principle deals with the supremum process and is an essential tool in its study. However the passage from the supremum to the terminal value is quite complicated and in general we should not hope that optimal inequalities for the terminal value could be obtained from the ones for supremum.

Acknowledgment. Author wants to express his gratitude towards professors Goran Peskir and Marc Yor for their help and support.

\section{References}

[1] J. Azéma and M. Yor. Le problème de Skorokhod: compléments à "Une solution simple au problème de Skorokhod". In Séminaire de Probabilités, XIII (Univ. Strasbourg, Strasbourg, 1977/78), volume 721 of Lecture Notes in Math., pages 625-633. Springer, Berlin, 1979.

[2] J. Azéma and M. Yor. Une solution simple au problème de Skorokhod. In Séminaire de Probabilités, XIII (Univ. Strasbourg, Strasbourg, 1977/78), volume 721 of Lecture Notes in Math., pages 90-115. Springer, Berlin, 1979.

[3] A. Cox and D. Hobson. An optimal Skorokhod embedding for diffusions. Stochastic Process. Appl., 111(1):17-39, 2004.

[4] B. Davis. On the $L^{p}$ norms of stochastic integrals and other martingales. Duke Math. J., 43(4):697-704, 1976.

[5] L. E. Dubins and G. Schwarz. A sharp inequality for sub-martingales and stopping-times. Astérisque, (157-158):129-145, 1988. Colloque Paul Lévy sur les Processus Stochastiques (Palaiseau, 1987).

[6] D. Gilat. On the ratio of the expected maximum of a martingale and the $L_{p}$-norm of its last term. Israel J. Math., 63(3):270-280, 1988.

[7] S. D. Jacka. Doob's inequalities revisited: a maximal $H^{1}$-embedding. Stochastic Process. Appl., 29(2):281-290, 1988.

[8] S. Karlin and H. M. Taylor. A second course in stochastic processes. Academic Press Inc. [Harcourt Brace Jovanovich Publishers], New York, 1981.

[9] I. Meilijson. The time to a given drawdown in Brownian motion. In Seminar on probability, XXXVII, volume 1832 of Lecture Notes in Math., pages 94-108. Springer, Berlin, 2003.

[10] J. Obłój. The Skorokhod problem and its offspring. Technical Report 886, LPMA - University of Paris 6.

[11] J. L. Pedersen and G. Peskir. Solving non-linear optimal stopping problems by the method of time-change. Stochastic Anal. Appl., 18(5):811-835, 2000.

[12] G. Peskir. The best Doob-type bounds for the maximum of Brownian paths. In High dimensional probability (Oberwolfach, 1996), volume 43 of Progr. Probab., pages 287-296. Birkhäuser, Basel, 1998.

[13] G. Peskir. Optimal stopping of the maximum process: the maximality principle. Ann. Probab., 26(4):1614-1640, 1998. 
[14] G. Peskir. Designing options given the risk: the optimal Skorokhodembedding problem. Stochastic Process. Appl., 81(1):25-38, 1999.

[15] G. Peskir. Maximum process problems in optimal control theory. Technical Report 423, Dept. Theoret. Statist. Aarhus, 2001.

[16] G. Peskir. A true Buyer's risk and classification of options. ITEM. Inf. Technol. Econ. Manag., (1):Paper 1, 20 pp. (electronic), 2001. 